\title{
Prevalencia de síndrome metabólico y relación con eventos cardiovasculares, supervivencia y función del injerto en pacientes con trasplante renal
}

\author{
Paola K. García ${ }^{a, b, *}$, Juan S. Gélvez ${ }^{a}$, Karen Navarro ${ }^{a}$, Kateir Contreras ${ }^{a, b}$, \\ Martha P. Rodríguez ${ }^{a, b}$ y Camilo González ${ }^{a, b}$
}

a Pontificia Universidad Javeriana, Facultad de Medicina, Bogotá, Colombia

${ }^{\mathrm{b}}$ Hospital Universitario San Ignacio, Servicio de Medicina Interna, Bogotá, Colombia

Recibido el 29 de noviembre de 2016; aceptado el 24 de mayo de 2017

Disponible en Internet el 31 de agosto de 2017

\section{PALABRAS CLAVE}

Síndrome metabólico; Supervivencia del injerto;

Trasplante renal

\begin{abstract}
Resumen
Introducción: el síndrome metabólico en pacientes trasplantados tiene una prevalencia entre 20 a $65 \%{ }^{1}$.

Objetivo: estimar la prevalencia de síndrome metabólico al momento del trasplante renal y su asociación con el desarrollo de eventos cardiovasculares, supervivencia del paciente y pérdida del injerto.

Métodos: estudio de cohorte retrospectiva, en el que se incluyeron pacientes con transplante renal entre los años 2004 y 2014. Se evaluó la asociación entre síndrome metabólico previo al trasplante y el desenlace compuesto de eventos cardiovasculares, pérdida del injerto y muerte por cualquier causa. Se efectuó un análisis univariado y multivariado tomando como significancia estadística una $\mathrm{p}<0,05$.

Resultados: se incluyeron 185 pacientes. La prevalencia del síndrome metabólico al momento del trasplante fue de 39\%; en el análisis univariado no se encontró una asociación significativa entre la presencia de síndrome metabólico al momento del trasplante y el desenlace compuesto de eventos cardiovasculares, pérdida del injerto o muerte por cualquier causa (RR 1,81, IC 95\% $0,86-3,82, p=0,11)$. Las variables relacionadas con el desenlace compuesto fueron diabetes mellitus $(O R=3,62$ IC 95\% 1,084-12,1 $\mathrm{p}=0,026)$ y tiempo de trasplante renal $(O R=1,05$ IC 95\% 1,007-1,09 $\mathrm{p}=0,36)$.

Conclusiones: no hubo una asociación significativa entre el síndrome metabólico al momento del trasplante renal y el desarrollo de eventos cardiovasculares, pérdida del injerto y muerte por cualquier causa. Esto puede estar relacionado con la baja incidencia de eventos en nuestra población y el tiempo de seguimiento.

(c) 2017 Sociedad Colombiana de Cardiología y Cirugía Cardiovascular. Publicado por Elsevier España, S.L.U. Este es un artículo Open Access bajo la licencia CC BY-NC-ND (http:// creativecommons.org/licenses/by-nc-nd/4.0/).
\end{abstract}

\footnotetext{
* Autor para correspondencia.

Correo electrónico: pkgarcia@husi.org.co (P.K. García).
} 


\section{KEYWORDS}

Metabolic syndrome;

Graft survival;

Kidney transplant

\section{Introducción}

El trasplante renal es la terapia de elección en pacientes con enfermedad renal crónica en estadio $5^{2}$. Provee mejor calidad de vida y supervivencia de los pacientes, en comparación con aquellos que se encuentran en diálisis ${ }^{3}$; sin embargo, aunque mejora las anormalidades relacionadas con la uremia se asocia con exposición a nuevos factores de riesgo (cambios en el estilo de vida, hábitos nutricionales y medicamentos) que predisponen al desarrollo de síndrome metabólico ${ }^{4}$. Adicionalmente, estos pacientes tienen factores de riesgo cardiovasculares como obesidad central, hipertensión arterial, dislipidemia y/o alteraciones en el metabolismo de la glucosa que favorecen la aterosclerosis ${ }^{5}$. El mecanismo fisiopatológico incluye resistencia a la insulina, bajo grado de inflamación crónica y deficiencia de ciertos elementos hormonales y nutricionales como 25 hidroxivitamina $D$ y adiponectina ${ }^{6}$.

En los pacientes trasplantados aumenta el riesgo de eventos cardiovasculares mayores y además se relaciona con disminución de la supervivencia y disfunción crónica del injerto ${ }^{7}$. A su vez, los componentes individuales del síndrome metabólico, especialmente la obesidad y la hipertensión arterial, también tienen efectos deletéreos en los desenlaces del trasplante renal $^{8,9}$. La diabetes mellitus de novo es una de las principales causas de morbilidad y mortalidad postrasplante, por tanto, las medidas dirigidas a reducir su incidencia tienen el potencial de mejorar los desenlaces en trasplante renal ${ }^{2}$.
De acuerdo con estudios previos, la prevalencia del síndrome metabólico en pacientes trasplantados renales oscila entre un $20 \%$ a un $65 \%$, y varía de acuerdo con la población estudiada y el tiempo de seguimiento postrasplante ${ }^{1}$. En consecuencia, el objetivo de este estudio fue determinar la prevalencia de síndrome metabólico al momento del trasplante y la asociación con eventos cardiovasculares y supervivencia del injerto y del paciente, información útil para implementar estrategias de seguimiento y tratamiento dirigidas a aquellos con mayor riesgo, en pro de disminuir la morbimortalidad.

\section{Materiales y métodos}

Se llevó a cabo un estudio de cohorte retrospectiva de pacientes trasplantados entre junio de 2004 y enero de 2014, en la que se incluyeron pacientes mayores de 18 años y se excluyeron aquellos que fallecieron o perdieron el injerto en los primeros tres meses del trasplante y que tuvieran trasplante combinado de órganos.

Se recolectaron datos de las historias clínicas y se obtuvieron variables demográficas como edad, género, talla, peso, índice de masa corporal (IMC) y variables clínicas como perfil de lípidos, glucosa en ayunas y presión arterial, así como tratamiento farmacológico para diabetes mellitus, hipertensión arterial y dislipidemia pre- y postrasplante. La definición de síndrome metabólico se tomó de una versión adaptada del National Cholesterol Education Expert Panel 
(Adult Treatment Panel III-ATP III) ${ }^{10}$, dado que el perímetro abdominal no se obtuvo en todos los pacientes y fue sustituido por el IMC ya que en estudios previos se ha visto que este cambio tiene pocos efectos en la aplicabilidad de la definición ${ }^{1,11}$. Los eventos cardiovasculares mayores se definieron como infarto agudo de miocardio, angina estable, angina inestable y evento cerebrovascular. La pérdida del injerto se definió como la necesidad de reiniciar diálisis o requerir otro trasplante. Se calculó la tasa de filtración glomerular (eTFG) según la ecuación MDRD 4.

Se efectuó un análisis bivariado del factor de exposición síndrome metabólico al momento del trasplante renal y mortalidad, pérdida del injerto y eventos cardiovasculares con el estadístico Chi cuadrado o $\mathrm{F}$ de Fisher, según correspondiera. Para variables cuantitativas como función renal se realizó un análisis de asociación bivariado con prueba $T$ Student o pruebas no paramétricas teniendo como variable dependiente síndrome metabólico. Para el ajuste de covariables y determinación de asociación ajustada entre síndrome metabólico y función renal se hizo un análisis de regresión lineal múltiple para la variable filtración glomerular, así como un ajuste de riesgo mediante regresión de Cox para el desenlace compuesto (mortalidad por cualquier causa, evento cardiovascular no fatal y pérdida del injerto) a fin de determinar el riesgo ajustado entre síndrome metabólico.

\section{Resultados}

Se incluyeron 185 pacientes con seguimiento promedio de 68,5 meses ( $\pm 11,9$ meses), receptores de donante cadavérico en el $95 \%$ de los casos. Las características demográficas y clínicas se incluyen en la tabla 1. La edad promedio al momento del trasplante fue de 45,1 años $( \pm 11,9$ años), 65,4\% fueron hombres. El tiempo promedio de diálisis previo al trasplante fue de 69,4 meses y la causa de enfermedad renal más frecuente fue hipertensión arterial $(26,5 \%)$, seguido de causa idiopática $(25,4 \%)$, glomerulonefritis $(18,3 \%)$ y diabetes mellitus $(12,9 \%)$. En cuanto al manejo farmacológico al año de trasplante $91,3 \%$ de los pacientes recibían inhibidores de calcineurina y $11,5 \%$ inhibidores de mTOR, cifras que pasaron a ser de $80 \%$ y $19,4 \%$ respectivamente a los tres años postrasplante.

Se halló una prevalencia de síndrome metabólico previo al trasplante renal de 39,4\%, que al año a los 3 años de seguimiento aumentó al 46 y al 55\%, respectivamente. En la tabla 2 se muestran los porcentajes de cada uno de los componentes del síndrome metabólico; $85,4 \%$ de los pacientes tenían diagnóstico de hipertensión arterial y $14 \%$ eran diabéticos. El promedio de IMC al momento del trasplante fue de $23,8 \mathrm{~kg} / \mathrm{m}^{2}$, lo cual aumentó de forma significativa al año (promedio IMC de $24,7 \mathrm{~kg} / \mathrm{m}^{2}, \mathrm{p}=0,015$ ) y a los 3 años (promedio IMC de $25,47 \mathrm{~kg} / \mathrm{m}^{2}, \mathrm{p}=0,0002$ ).

Al analizar el desenlace primario no se encontró una asociación significativa entre la presencia de síndrome metabólico al momento del trasplante y el desenlace compuesto de eventos cardiovasculares, pérdida del injerto o muerte por cualquier causa (RR 1,81, IC 95\% 0,86-3,82, $\mathrm{p}=0,11)$. Al ajustar el riesgo por edad, sexo, IMC, tiempo de trasplante, tiempo en diálisis, comorbilidad, inmunosupresor usado y paraclínicos tampoco se encontró asociación de
Tabla 1 Características clínicas y demográficas basales de la población trasplantada

\begin{tabular}{|c|c|}
\hline Variable & Pacientes (185) \\
\hline $\begin{array}{l}\text { Sexo } \\
\text { - Femenino } \\
\text { - Masculino }\end{array}$ & $\begin{array}{l}64(34,5 \%) \\
121(65,4 \%)\end{array}$ \\
\hline $\begin{array}{l}\text { Edad (años) } \\
\text { - Promedio } \\
\text { Donante } \\
\text { - Cadavérico }\end{array}$ & $\begin{array}{l}45,1(\mathrm{DE} \pm 11,9) \\
176(95,1 \%)\end{array}$ \\
\hline $\begin{array}{l}\text { Etiología ERC } \\
\text { - HTA } \\
\text { - Idiopática } \\
\text { - Glomerulonefritis } \\
\text { - Diabetes } \\
\text { - Enfermedad poliquística } \\
\text { - Nefrotoxicidad } \\
\text { - Litiasis renal } \\
\text { - Síndrome de Alport } \\
\text { - LES }\end{array}$ & $\begin{array}{l}49(26,4 \%) \\
47(25,4 \%) \\
34(18,3 \%) \\
24(12,9 \%) \\
9(4,8 \%) \\
3(1,6 \%) \\
2(1,08 \%) \\
4(2,16 \%) \\
3(1,6 \%)\end{array}$ \\
\hline $\begin{array}{l}\text { Peso (kg) } \\
\text { - Promedio }\end{array}$ & $67,6(\mathrm{DE} \pm 11,09)$ \\
\hline $\begin{array}{l}\text { Talla (metros) } \\
\text { - Promedio }\end{array}$ & $1,63(\mathrm{DE} \pm 0,93)$ \\
\hline $\begin{array}{l}\text { IMC }\left(\mathrm{kg} / \mathrm{m}^{2}\right) \\
\text { - Promedio }\end{array}$ & $23,8(\mathrm{DE} \pm 2,92)$ \\
\hline $\begin{array}{l}\text { Diabetes mellitus } \\
\text { - Si } \\
\text { - No }\end{array}$ & $\begin{array}{l}26(14,05 \%) \\
159(85,9 \%)\end{array}$ \\
\hline $\begin{array}{l}\text { Hipertensión arterial } \\
\text { - Si } \\
\text { - No }\end{array}$ & $\begin{array}{l}158(85,4 \%) \\
27(14,5 \%)\end{array}$ \\
\hline $\begin{array}{l}\text { Síndrome metabólico } \\
\text { - Si } \\
\text { - No }\end{array}$ & $\begin{array}{l}73(39,4 \%) \\
112(60,5 \%)\end{array}$ \\
\hline
\end{tabular}

ERC: enfermedad renal crónica, HTA: hipertensión arterial, IMC: índice de masa corporal, DS: desviación estándar. LES: lupus eritematoso sistémico.

síndrome metabólico con el desenlace compuesto $(p=0,98)$. Las variables relacionadas con el desenlace compuesto fueron diabetes mellitus al trasplante $(O R=3,62$ IC $95 \%$ $1,084-12-1 p=0,026)$ y tiempo de trasplante renal $(O R=1,05$ IC95\% 1,007-1,09 $\mathrm{p}=0,36$ ).

Durante el tiempo de seguimiento hubo en total 16 eventos cardiovasculares, de los cuales 9 se presentaron en pacientes con síndrome metabólico al momento del trasplante versus 7 en pacientes sin síndrome metabólico, sin hallarse una diferencia estadísticamente significativa entre ambos grupos en el modelo univariado $(12,3 \%$ vs. $6,2 \%$, $\mathrm{p}$ : NS) ni en el multivariado. La incidencia de eventos cardiovasculares fue de $2,2 \%$ en el primer año y de $3,7 \%$ a los 3 años del trasplante (tabla 3 ).

En cuanto a la función del injerto renal al año de seguimiento, no hubo diferencias significativas en la eTFG por MDRD4 en pacientes sin síndrome metabólico y con síndrome 
Tabla 2 Comportamiento del síndrome metabólico

\begin{tabular}{|c|c|c|c|c|}
\hline Variable & Pretrasplante & 1 año & 3 años & $\mathrm{p}$ \\
\hline Síndrome metabólico & $39,4 \%$ & $46,9 \%$ & $55,6 \%$ & $<0,05$ \\
\hline Hiperglicemia & $23,2 \%$ & $31,6 \%$ & $32,5 \%$ & $<0,05$ \\
\hline Diabetes Mellitus & $14,0 \%$ & $14,4 \%$ & $7,5 \%$ & \\
\hline Hipertensión arterial & $85,4 \%$ & $86,2 \%$ & $85 \%$ & NS \\
\hline Índice de masa corporal & $23,8 \mathrm{~kg} / \mathrm{m}^{2}$ & $24,7 \mathrm{~kg} / \mathrm{m}^{2}$ & $25,5 \mathrm{~kg} / \mathrm{m}^{2}$ & $<0,05$ \\
\hline Peso & $63,5 \mathrm{~kg}$ & $65,8 \mathrm{~kg}$ & $67,6 \mathrm{~kg}$ & \\
\hline Triglicéridos elevados & $55,1 \%$ & $63,8 \%$ & $63,1 \%$ & $<0,05$ para 1 er. año \\
\hline HDL bajo & $56,2 \%$ & $64,1 \%$ & $63,1 \%$ & $<0,05$ para 1 er. año \\
\hline
\end{tabular}

Tabla 3 Eventos cardiovasculares en población con síndrome metabólico

\begin{tabular}{llll}
\hline $\begin{array}{l}\text { Síndrome } \\
\text { metabólico }\end{array}$ & 1 año & 3 años & $\begin{array}{l}\text { Total al final } \\
\text { seguimiento }\end{array}$ \\
\hline $\mathrm{Si}$ & 1 & 2 & $9(12,3 \%)$ \\
No & 3 & 5 & $7(6,2 \%)$ \\
Total & $4(2,2 \%)$ & $7(3,7 \%)$ & 16 \\
\hline
\end{tabular}

metabólico $(63,35 \pm 1,59 \mathrm{ml} / \mathrm{min}$ vs. $64,95 \pm 2,08 \mathrm{ml} / \mathrm{min}$ con $p=0,27)$, ni en proteinuria $(p=0,61)$ en asociación con el diagnóstico de síndrome metabólico al momento del trasplante renal. Tampoco hubo diferencias significativas en estas variables a los tres años postrasplante $(p=0,16$ y $p=0,53$ respectivamente).

\section{Discusión}

Se analizaron las características demográficas y clínicas de la cohorte de pacientes en los primeros 10 años del programa de trasplante renal y se halló como principal causa de enfermedad renal estadio 5, la hipertensión arterial, la cual es uno de los componentes del síndrome metabólico, que se define como un conjunto de factores de riesgo cardiovascular que, en pacientes con trasplante, aumenta el riesgo de desenlaces desfavorables y la disfunción crónica del injerto y disminuye la sobrevida del mismo ${ }^{7}$.

Si bien la prevalencia del síndrome metabólico en los pacientes con trasplante renal puede estar entre el 20 al $65 \%$ según diferentes estudios ${ }^{1}$, la prevalencia del síndrome metabólico pretrasplante en la cohorte estudiada fue mayor a la reportada en estudios como los de Oruc o Bonet $^{12,13}$, que fue $34,2 \%$ y $25,8 \%$ respectivamente. Similar a lo descrito por LaGuardia et al., tanto la prevalencia del síndrome metabólico, como la de sus componentes individuales, a excepción de la hipertensión arterial, aumentaron en el periodo postrasplante ${ }^{14}$. Esto se explica por cambios en el estilo de vida, hábitos nutricionales y medicamentos incluyendo corticosteroides, inhibidores de la calcineurina e I-mTOR. En una cohorte prospectiva de 71.527 pacientes con trasplante de órgano sólido, el sobrepeso y la obesidad estuvieron asociados con aumento del riesgo de infarto de miocardio y enfermedad coronaria independiente del síndrome metabólico ${ }^{15}$. La obesidad pretrasplante definida como IMC > 30 se asoció con incremento del riesgo de fun- ción retardada del injerto (RR, 1,41; IC 95\% 1,26-1,57) pero no con rechazo agudo ${ }^{16}$.

La hiperglicemia y la diabetes mellitus postrasplante tienen una prevalencia del 2 al 53\% de acuerdo con la definición usada. La incidencia estimada en Estados Unidos es 9,1\% a tres meses, $16 \%$ a once meses y $24 \%$ a 36 meses $^{2}$. Otros estudios muestran que la incidencia de diabetes mellitus postrasplante es del 13 al $43 \%$ en los primeros cinco años ${ }^{17,18}$. En la cohorte estudiada la prevalencia de diabetes mellitus en el momento del trasplante fue de $14 \%$ y aumentó a $17 \%$ a los 36 meses del seguimiento.

Pese a ello no se encontró asociación estadísticamente significativa entre síndrome metabólico al momento del trasplante con la aparición de eventos cardiovasculares, función del injerto ni muerte por cualquier causa, hecho que puede estar asociado con la baja incidencia de eventos cardiovasculares en esta cohorte como también con la identificación precoz de pacientes con mayor riesgo cardiovascular y la instauración temprana de medidas farmacológicas y no farmacológicas. No se descarta que con un tiempo de seguimiento más prolongado y con un mayor tamaño de muestra, pueda encontrarse asociación.

Aunque el trasplante renal disminuye el riesgo de enfermedad cardiovascular en comparación con pacientes en diálisis, el riesgo anual de eventos cardiovasculares y mortalidad resulta 10 a 50 veces más alto que en la población general ${ }^{19}$, y la prevención de las complicaciones del síndrome metabólico posterior al trasplante renal es un factor importante para mantener el injerto y disminuir la morbimortalidad $^{20}$. Por esto, aunque no encontrara una asociación significativa entre el síndrome metabólico y los desenlaces preestablecidos, este estudio es de utilidad en el conocimiento de la epidemiología y el comportamiento de las alteraciones metabólicas en nuestra población.

\section{Conclusión}

El síndrome metabólico es altamente prevalente en receptores de trasplante renal; en la población estudiada alcanzó un $39 \%$, cifra incluso mayor comparada con otros estudios. Sin embargo, no se encontró asociación estadísticamente significativa con eventos cardiovasculares, función del injerto ni muerte por cualquier causa durante el seguimiento, lo cual puede estar asociado con la baja incidencia de eventos cardiovasculares y el tiempo de seguimiento. Hubo un aumento significativo en el IMC en el postrasplante, y con ello incremento en la prevalencia de síndrome metabólico luego de 
tres años. Se recomienda educar al paciente respecto a su nutrición, promover hábitos de vida saludable e instaurar medidas farmacológicas según sea el caso.

\section{Responsabilidades éticas}

Protección de personas y animales. Los autores declaran que para esta investigación no se han realizado experimentos en seres humanos ni en animales.

Confidencialidad de los datos. Los autores declaran que han seguido los protocolos de su centro de trabajo sobre la publicación de datos de pacientes.

Derecho a la privacidad y consentimiento informado. Los autores declaran que en este artículo no aparecen datos de pacientes.

\section{Financiación}

Ninguna.

\section{Conflictos de interés}

Ninguno.

\section{Bibliografía}

1. Porrini E, Delgado P, Bigo C, Alvarez A, Cobo M, Checa M. Agregar hasta seis autores Impact of metabolic syndrome on graft function and survival after cadaveric renal transplantation. Am J Kidney Dis. 2006;48:134-42.

2. Sneha P, Ramesh GV. New-onset diabetes mellitus after kidney transplantation: Current status and future directions. World J Diabetes. 2015;6:445-55.

3. Shirali AC, Bia MJ. Management of cardiovascular disease in renal transplant recipients. Clin J Am Soc Nephrol. 2008;3:491-504.

4. Niemirska LM. Metabolic syndrome in children with chronic kidney disease an after renal transplantation. Pediatr Nephrol. 2014;29:203-16.

5. Ford ES, Li C, Sattar N. Metabolic syndrome and incident diabetes: current state of the evidence. Diabetes Care. 2008;31:1898-904.

6. Kulshrestha S, Ojo A, Luan Fu. Metabolic syndrome, vitamin d deficiency and hypoadiponectinemia among non-diabetic patients early after kidney transplantation. Am J Nephrol. 2013;37:399-404.

7. Houri Inbal, Tzukert K, Levi Irit, Aharon M, Bloch Aharon, Gotsman O. Implementation of guidelines for metabolic syndrome control in kidney transplant recipients: results at single center. Diabetol Metab Syndr. 2015;7:90-9.

8. Chow KM, Szeto CC, Leung CB, Lui SF, Tong YF, Kam-Tao Li P. Body mass index as a predictive factor for long-term renal transplant outcomes in Asians. Clin Transplant. 2005;20:582-9.

9. De Vries A, Bakker S, van Son W, Homan van der Heide J, Hauw $\mathrm{T}$, Jong $\mathrm{P}$, et al. Insulin resistance as a putative cause of chronic renal transplant dysfunction. Am J Kidney Dis. 2003;41:859-67.

10. Lorenzo C, Williams K, Hunt K, Haffner S. The National Cholesterol Education Program Adult Treatment Panel III, International diabetes Federation, and World Health Organization Definitions of The Metabolic Syndrome as Predictors of Incident Cardiovascular Disease and Diabetes. Diabetes Care. 2007;30:8-13.

11. Meigs J, Wilson P, Nathan D, D’Agostino R, Williams K, Haffner S. Prevalence and characteristics of the metabolic syndrome in the San Antonio and Framingham off-spring studies. Diabetes. 2003;52:2160-7.

12. Oruc M, Koseoglu K, Seyahi N, Alagoz S, Trabulus S, Altiparmark MR. Progression of metabolic syndrome in renal transplant recipients. Transplant Proc. 2013;45:3273-8.

13. Bonet J, Martinez A, Bayés B. Metabolic syndrome in hemodialysis patients as a risk factor for new-onset diabetes mellitus after renal transplant: a prospective observational study. Diabetes, Metabolic Syndrome and Obesity: Targets and Therapy. 2013;6:339-46.

14. LaGuardia H, Zhang R. Obesity and metabolic syndrome in kidney transplantation. Curr Hypertens Rep. 2013;15:215-23.

15. Thomsen M, Nordestgaard BG. Myocardial infarction and ischemic heart disease in overweight and obesity with a without metabolic syndrome. JAMA. 2014;174:15-22.

16. Beckmann S, Ivanovic N, Drent G, Ruppar T, De Geest S. Weight gain, overweight and obesity in solid organ transplantation-a study protocol for a systematic literature review. Systematic Reviews. 2015;4:2-10.

17. Israni A, Snyder J, Skeans MA, Kasiske B. Clinical diagnosis of metabolic syndrome: predicting new onset diabetes, coronary heart disease, and allograft failure after kidney transplant. Transpl Int. 2012;25:748-51.

18. Bayer ND, Cochetti PT, Kumar A, Teal V, Huan Y, Doria C, et al. Association of metabolic syndrome with development of new-onset diabetes after transplantation. Transplantation. 2010;90:861-6.

19. Ojo AO. Cardiovascular complications after renal transplantation and their prevention. Transplantation. 2006;82:603-11.

20. Moriyama T, Takahara S. Kidney transplantation and metabolic syndrome. Clinic All-Round. 2006;55:2032-5. 